\title{
Optimal Relay Selection and Power Allocation in Cooperative Cognitive Communication System
}

\author{
Guanglong Yang ${ }^{1,2}$, Xiao Wang ${ }^{1,2}$ and Xuezhi Tan ${ }^{1,2}$ \\ ${ }^{1}$ Communication Research Center, Harbin Institute of Technology, Harbin, 150080, \\ China \\ ${ }^{2}$ Key Laboratory of Police Wireless Digital Communication, Ministry of Public \\ Security, Harbin, 150080, China
}

hit12b@126.com; hitwx@hit.edu.cn; tanxz1957@hit.edu.cn

\begin{abstract}
This paper focuses on the optimal relay selection and power allocation issue, which studies the communication between SS (Secondary Source) and two SDs (Secondary Destinations) through single relay in $C R$ (cooperative cognitive communication) system. Sufficiently takes the multi-antenna relay, primary-secondary users coexisting, and AF (Amplify and Forward) relay mode as the researching background, and takes the constraints caused by the primary user's interference and the max transmission power into account, the author proposes to maximize the system throughput by the selection of cooperative relay and the allocation of optimal power. Furthermore, the author also puts forward the approximate expressions of the optimal power allocation, and figures out the optimal solution by the Lagrange multiplier, whose property can be manifested by the simulation and comparison results.
\end{abstract}

Keywords: cooperative cognitive communication systems, multi-antenna relay, relay selection, power allocation, system throughput

\section{Introduction}

CR (Cognitive radio) can solve the problem of spectrum resource scarcity by further allocating the granted spectrum [1]. Thus, how to allocate the spectrum efficiently to increase the cognitive users' communication, and improve the cognitive system throughput has become a hot issue in current research. Under CR underlay mode, cognitive users and the primary users may share the same spectrum, in order to avoid causing interference to the primary users, the cognitive users have to resort to lower transmission power [2-4].Thus, the application of relay technology is very necessary for the realization of cognitive users 'communication, it can play an important role in expanding the cognitive users 'communication range and improving the system throughput [5-6].

There is a common situation in cooperative cognitive communication system one single SS communicates with two or more SDs through the single relay broadcast. However, if the SDs are located too dispersive, or the distance from the relay are too long, the node with a longer distance from the relay may can't receive information efficiently, which is also an energy waste for the node with a shorter distance from the relay. So, in this paper the author proposes a new solution to solve the above mentioned problem. He takes the multi-antenna relay, which can make data transmission with different nodes under AF mode through orthogonal 
channel equipment, and takes the constraints caused by the primary user's "interference temperature "into account, and tries to figure out how to select the replay, and then how to allocate the transmission power of different channels.

The common relays are amplify-and-forward (AF) and Decode-and-forward (DF). In the AF mode, the relay node linear amplifies and forwards the received signal directly; In DF mode, the relay node decodes the received signal from the source node and then forwards to the SD after recoding. In literature review [7] the author puts forward a cognitive relay decode-and-forward transmission solution based on the selection of optimal relay, which maximizes the cognitive user's information transmission rate. Compared to AF mode, DF mode avoids the noise amplification of replay node, that increases the efficiency of power, but if there is a decoding error, it may bring out the diffusion error problems. So the research here is carried out under the AF mode in this paper.

How to select the appropriate relay is particularly important in cooperative communication. A great number of literatures have studied the allocation problem of cooperative relay power under the CR system. Literature [8] puts forward a cooperative transmission solution based on AF mode, it makes a theoretical analysis on the conflict-free transmission time, and figures out the analytical expressions of conflict-free transmission under the cooperative and noncooperative mode separately. Also, Literature [9] proposes a cognitive relay selection algorithm based on location and interference constraint, which selects the relay node according to the effect of the node location on the relay link rate and determines the optimal transmission power of relay nodes, under the "limiting interference to the primary users" circumstance. In Literature [10], the author puts forward the approximate expression of terminal probability by analyzing the cumulative distribution expression of end-to-end SNR, and makes a deep discussion about the cognitive network performance within the primary user's interference tolerance. Under AF mode, Literature [11] discusses about the optimal relay selection between one SS and one SD through multiple relay nodes. On the basic of channel state information (CSI) Literature [12] introduces two kinds of relay selection algorithm Minimum channel gain algorithm and Average channel gain algorithm. In traditional wireless communication, we often select replay according to if it can maximize system throughput. So the author also takes this as target to conduct optimal relay selection. In traditional relay system, we don' t take interference into account. While in CR system, we need to take the primary user' $\mathrm{s}$ interference tolerance into consideration. Thus, there is a great distinction between the relay selection algorithm of CR system and traditional system. After selecting the replay, the CR source node and the replay node transmission power should guarantee there is no interference to the primary user.

Literature [13] maximizes system throughput by utilizing search algorithm to select the relay, however, it doesn't take the primary users' interference into consideration. In literature [14] the author selects the free users as cooperative relay to forward the source node data of cognitive users, and finally he figures out the optimal and the sub-optimal relay selection algorithm to get the max throughput of the system. Under the mode of multi-user and single relay cognitive network in Literature [15], the author allocated the cognitive reply power with game mode, in order to balance the total network rate and users fairness. In literature [16] the author discussed the OFDM (Orthogonal Frequency Division Multiple Access) uplink relay selection, power allocation and the combination and optimization problems of subcarrier allocation, and furthermore, he realized the maximum system throughput by utilizing double dual decomposition method and sub-gradient method. In literature [17] the author discusses the resource allocation in single-user OFDM relay cooperative system. In this paper the author maximizes the system throughput by utilizing the twin carrier relay, which can receive data by one subcarrier, and forward data by another subcarrier. 
In this paper, sufficiently take the multi-antenna relay, primary-secondary users coexisting, and AF (Amplify and Forward) relay mode as the researching background, and take the constraints caused by the primary user's interference and the max transmitted power into account, the author proposes to maximize the system throughput by cooperative relay selection and optimal power allocation.

\section{System Model and Problem Formulation}

As shown in Figure 1, this paper is under the primary-secondary users coexisting background. There is one PU (primary user), one SS (secondary source), several R (relays), and two SD (secondary destination) in the CR system.

In this paper, we assume that the distance from SS and SD is too long to get the data directly. So the author utilizes the orthogonal channel for data transmission under the AF replay mode. After receiving and amplifying the data, the replay forwards it to two different SD by orthogonal channel $(\mathrm{CH}) 1$ and $(\mathrm{CH}) 2$.

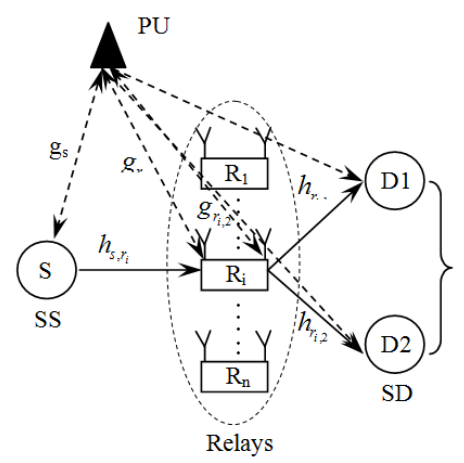

Figure 1. The Structure of Cooperative Cognitive Communication Systems

There are two time slots in the communication process in this paper. Within the first time slot, SS sends the data, and the replay receives it. Here we consider the source signal as $x \in \square \mathrm{E}\left\{|x|^{2}\right\}=1$, and the received signal is

$$
r_{i}=\sqrt{P_{s}} h_{s, r_{i}} x+n_{p, r_{i}}+n_{0}
$$

Here $P_{s}$ is the transmission power of SS, $h_{s, r_{i}}$ is the channel gain from SS and relay $R_{i}, n_{p, r_{i}}$ is the interference of primary user to replay $R_{i}, n_{0}$ is the Additive white Gaussian noise around the replay node, the mean value of white noise is 0 , and variance is $N_{0}$.

In the second time slot, the author adopts the same technique with Literature [14], he amplifies the data received in the first time slot, and forwards the data to SD $\mathrm{CH} 1$ and $\mathrm{CH} 2$ by orthogonal channel. The signal $P_{r_{i}, d_{1}}$ and $P_{r_{i}, d_{2}}$ received by SD are as follows

$$
\begin{aligned}
& P_{r_{i}, d_{1}}=\sqrt{p_{r_{i, 1}}} h_{r_{i, 1}}\left(\sqrt{p_{s}} h_{s, r_{i}} x+n_{p, r_{i}}+n_{0}\right)+n_{p, 1}+n_{0} \\
& P_{r_{i}, d_{2}}=\sqrt{p_{r_{i, 2}}} h_{r_{i, 2}}\left(\sqrt{p_{s}} h_{s, r_{i}} x+n_{p, r_{i}}+n_{0}\right)+n_{p, 2}+n_{0}
\end{aligned}
$$

$p_{r_{i, 1}}$ and $p_{r_{i, 2}}$ are the transmission power of replay $R_{i}$ on the orthogonal channel CH1and $\mathrm{CH} 2, h_{r_{i, 1}}$ and $h_{r_{i, 2}}$ are the channel gain from replay $R_{i}$ to SD D1 and D2 respectively. $n_{p, 1}$ and 
$n_{p, 2}$ are the interference of the primary user to SDs respectively, and $n_{0}$ is the Additive white Gaussian noise around the replay node.In this paper, we assume the SD as single-antenna relay. And the SD 1 and SD2 regard the information for each other as interference; SDs can easily filter this interference according to channel knowledge. So there is no reflection of this interference in (2 a) and (2 b).After normalization, the SNRs of D1and are as follows separately.

$$
\begin{aligned}
& \operatorname{SINR}_{s, d_{1}}=\frac{p_{s} p_{r_{i, 1}}\left|h_{s, r_{i}}\right|^{2}\left|h_{r_{i, 1}}\right|^{2}}{p_{r_{i, 1}}\left|h_{r_{i, 1}}\right|^{2}\left(N_{p, r_{i}}+N_{0}\right)+\left(N_{p, 1}+N_{0}\right)} \\
& \operatorname{SINR}_{s, d_{2}}=\frac{p_{s} p_{r_{i, 2}}\left|h_{s, r_{i}}\right|^{2}\left|h_{r_{i, 2}}\right|^{2}}{p_{r_{i, 2}}\left|h_{r_{i, 2}}\right|^{2}\left(N_{p, r_{2}}+N_{0}\right)+\left(N_{p, 2}+N_{0}\right)}
\end{aligned}
$$

CR system should take the interference of primary user into consideration, in the first time slot, the transmission power of SS meets the interference tolerance, which would lead to the interference of primary user. The transmission power of SS should satisfy

$$
p_{s}\left|g_{s}\right|^{2} \leq I_{\max }
$$

$I_{\max }$ is the interference tolerance of primary user, similarly, in the second time slot, the transmission power of relay $i$ should meet the following condition

$$
\begin{aligned}
& p_{i_{1}}\left|g_{r_{i, 1}}\right|^{2} \leq I_{1} \\
& p_{i_{2}}\left|g_{r_{i, 2}}\right|^{2} \leq I_{2}
\end{aligned}
$$

$I_{1}$ and $I_{2}$ are the interference tolerance of primary user on channel 1 and channel 2, which satisfies the following condition

$$
I_{1}+I_{2} \leq I_{\max }
$$

In the above formula, $g_{s}$ is the channel gain from SS to primary user, $g_{r_{i, 1}}$ and $g_{r_{i, 2}}$ are channel gains from replay $R_{i}$ to primary user through $\mathrm{CH} 1$ and $\mathrm{CH} 2$.

The transmission power of SS is limited by the interference tolerance of primary user and the max transmission power itself.

$$
p_{s} \leq p_{s \max }
$$

$p_{s \max }$ is max transmission power of SS, and the transmission power of replay $R_{i}$ should meet the following requirement.

$$
p_{r_{i, 1}}+p_{r_{i, 2}} \leq p_{r_{\text {max }}}
$$

$p_{r \text { max }}$ is the max transmission power of replay $R_{i}$. 


\section{Optimal Relay Selection and Power Allocation}

\subsection{Algorithm-oriented Mathematical Models}

This chapter specifies optimal relay selection and power allocation algorithm with a view to selecting maximum system throughput of optimal relay $R_{i}$. Provided that $T_{r_{i}}\left(P_{s}, P_{r_{i, 1}}, P_{r_{i, 2}}\right)$ is instantaneous total throughput after the relay $R_{i}$ is selected, the expression is shown as follow

$$
\begin{gathered}
T_{r_{i}}\left(P_{s}, P_{r_{i, 1}}, P_{r_{i, 2}}\right)=\frac{1}{2} \log \left(1+\operatorname{SINR}_{s, d_{1}}\right)+\frac{1}{2} \log \left(1+\operatorname{SINR}_{s, d_{2}}\right) \\
\approx \frac{1}{2} \log \left(\frac{p_{s} p_{r_{i, 1}}\left|h_{s, r_{i}}\right|^{2}\left|h_{r_{i, 1}}\right|^{2}}{p_{r_{i, 1}}\left|h_{r_{i, 1}}\right|^{2}\left(N_{p, r_{i}}+N_{0}\right)+\left(N_{p, 1}+N_{0}\right)}\right)+\frac{1}{2} \log \left(\frac{p_{s} p_{r_{i, 2}}\left|h_{s, r_{i}}\right|^{2}\left|h_{r_{i, 2}}\right|^{2}}{p_{r_{i, 2}}\left|h_{r_{i, 2}}\right|^{2}\left(N_{p, r_{i}}+N_{0}\right)+\left(N_{p, 2}+N_{0}\right)}\right) \\
=\frac{1}{2} \log \left(\frac{p_{s}^{2} p_{r_{i, 1}} p_{r_{i, 2}}\left|h_{s, r_{i}}\right|^{4}\left|h_{r_{i, 1}}\right|^{2}\left|h_{r_{i, 2}}\right|^{2}}{\left[p_{r_{i, 1}}\left|h_{r_{i, 1}}\right|^{2}\left(N_{p, r_{i}}+N_{0}\right)+\left(N_{p, 1}+N_{0}\right)\right]\left[p_{r_{i, 2}}\left|h_{r_{i, 2}}\right|^{2}\left(N_{p, r_{i}}+N_{0}\right)+\left(N_{p, 2}+N_{0}\right)\right]}\right)
\end{gathered}
$$

In formula (6), provided that $A=p_{r_{i, 1}}\left|h_{r_{i, 1}}\right|^{2}\left(N_{p, r_{i}}+N_{0}\right)+\left(N_{p, 1}+N_{0}\right)$, $B=p_{r_{i, 2}}\left|h_{r_{i, 2}}\right|^{2}\left(N_{p, r_{i}}+N_{0}\right)+\left(N_{p, 2}+N_{0}\right)$, The formula (6) can be simplified as

$$
T_{r_{i}}\left(P_{s}, P_{r_{i, 1}}, P_{r_{i, 2}}\right)=\frac{1}{2} \log \left(\frac{p_{s}^{2} p_{r_{i, 1}} p_{r_{i, 2}}\left|h_{s, r_{i}}\right|^{4}\left|h_{r_{i, 1}}\right|^{2}\left|h_{r_{i, 2}}\right|^{2}}{A B}\right)
$$

Based on the discussion mentioned above, the issues specified in this article can be converted into optimal ones as follows to solve

$$
\begin{aligned}
\max _{p_{s}, p_{i}, p_{i_{2}}} & \frac{1}{2} \log \left(\frac{p_{s}^{2} p_{r_{i, 1}} p_{r_{i, 2}}\left|h_{s, r_{i}}\right|^{4}\left|h_{r_{i, 1}}\right|^{2}\left|h_{r_{i, 2}}\right|^{2}}{A B}\right) \\
\text { s.t. } & p_{s} \leq p_{s \max }, \\
& p_{r_{i, 1}}+p_{r_{i, 2}} \leq p_{r \max }, \\
& 0 \leq p_{s}\left|g_{s}\right|^{2} \leq I_{\max }, \\
& 0 \leq p_{i_{1}}\left|g_{r_{i, 1}}\right|^{2} \leq I_{1}, \\
& 0 \leq p_{i_{2}}\left|g_{r_{i, 2}}\right|^{2} \leq I_{2}, \\
& I_{1}+I_{2} \leq I_{\max }
\end{aligned}
$$

Known from formula (8), the objective function $T_{i}$ is a monotone increasing function with variable of $P_{s}$, the transmission power of $P_{s}$ is subject to the restraint of interference 
tolerance of primary users, its optimal value can be expressed as $P_{s}^{*}=\min \left(P_{s \max }, \frac{I_{1}}{\left|g_{s}\right|^{2}}\right)$; when optimal value of $P_{s}$ is acquired, the system maximum throughput can be achieved by means of calculating the optimal values $\left(P_{r_{i, 1}}, P_{r_{i, 2}}\right)$ with interference tolerance of primary users. Because of the increasing property of $T_{i}$ function, the maximization issue of the above formula can be equivalent to minimization issue

$$
\min _{p_{s}, p_{i_{1}}, p_{i_{2}}} \frac{A B}{\left(P_{s}^{*}\right)^{2} P_{r_{i, 1}} P_{r_{i, 2}}\left|h_{s, r_{i}}\right|^{4}\left|h_{r_{i, 1}}\right|^{2}\left|h_{r_{i, 2}}\right|^{2}}
$$

Known from the restraint conditions, $p_{r_{i, 1}}+p_{r_{i, 2}} \leq p_{r \max }, \quad p_{i_{1}}\left|g_{r_{i, 1}}\right|^{2}+p_{i_{2}}\left|g_{r_{i, 2}}\right|^{2} \leq I_{\max }$, provided that the value of $P_{r_{i, 1}}$ and $P_{r_{i, 2}}$ is gradually increased by relay $R_{i}$, the minimization issue of formula (9) can be discussed by means of the following two sub-methods.

\subsection{Power Allocation Algorithm}

\section{A. OMI (Optimal approach with Max Interference threshold) Algorithm}

OMI algorithm is conducting the optimal relay power allocation under the restraint condition of maximum interference tolerance of primary users; provided that $p_{r_{i, 1}}+p_{r_{i, 2}} \leq p_{r \max }, \quad p_{r_{i, 1}}\left|g_{r_{i, 1}}\right|^{2}+p_{r_{i, 2}}\left|g_{r_{i, 2}}\right|^{2}=I_{\max }$, namely, when the values of $P_{r_{i, 1}}$ and $P_{r_{i, 2}}$ gradually increase to the critical value of interference tolerance of primary user, they have not reached the maximum transmission power of relay of the cognitive user, therefore, the restraint condition can be simplified as $p_{r_{i, 1}}\left|g_{r_{i, 1}}\right|^{2}+p_{r_{i, 2}}\left|g_{r_{i, 2}}\right|^{2}=I_{\max }$.

By means of Lagrange multiplier, the optimal Lagrange function of OMI algorithm is

$$
L\left(P_{r_{i, 1}}, P_{r_{i, 2}}, \lambda\right)=\frac{A B}{\left(P_{s}^{*}\right)^{2} P_{r_{i, 1}} P_{r_{i, 2}}\left|h_{s, r_{i}}\right|^{4}\left|h_{r_{i, 1}}\right|^{2}\left|h_{r_{i, 2}}\right|^{2}}+\lambda\left(p_{r_{i, 1}}\left|g_{r_{i, 1}}\right|^{2}+p_{r_{i, 2}}\left|g_{r_{i, 2}}\right|^{2}-I_{\max }\right)
$$

Among which, $\lambda$ is Lagrange multiplicator, and the expressions can be obtained

$$
\begin{gathered}
\frac{\partial L}{\partial P_{r_{i, 1}}}=\frac{\partial\left(\frac{A B}{\left(P_{s}^{*}\right)^{2} P_{r_{i, 1}} P_{r_{i, 2}}\left|h_{s, r_{i}}\right|^{4}\left|h_{r_{i, 1}}\right|^{2}\left|h_{r_{i, 2}}\right|^{2}}\right)}{\partial P_{r_{i, 1}}}+\lambda\left|g_{r_{i, 1}}\right|^{2}=0 \\
\frac{\partial L}{\partial P_{r_{i, 2}}}=\frac{\partial\left(\frac{A B}{\left(P_{s}^{*}\right)^{2} P_{r_{i, 1}} P_{r_{i, 2}}\left|h_{s, r_{i}}\right|^{4}\left|h_{r_{i, 1}}\right|^{2}\left|h_{r_{i, 2}}\right|^{2}}\right)}{\partial P_{r_{i, 2}}}+\lambda\left|g_{r_{i, 2}}\right|^{2}=0 \\
p_{r_{i, 1}}\left|g_{r_{i, 1}}\right|^{2}+p_{r_{i, 2}}\left|g_{r_{i, 2}}\right|^{2}=I_{\max }
\end{gathered}
$$


Provided the variable $a=N_{p, r_{i}}+N_{0}, \quad b=N_{p, 1}+N_{0}, \quad c=N_{p, 2}+N_{0}$

$$
d_{1}=\sqrt{\left(I_{\max }\left|h_{r_{i, 2}}\right|^{2} a b+\left|g_{r_{i, 2}}\right|^{2} b c\right)^{2}-\left(\left|g_{r_{i, 1}}\right|^{2}\left|h_{r_{i, 2}}\right|^{2} a b-\left|h_{r_{i, 1}}\right|^{2}\left|g_{r_{i, 2}}\right|^{2} a c\right)\left(I_{\max }^{2}\left|h_{r_{i, 2}}\right|^{2} a b+I_{\max }\left|g_{r_{i, 2}}\right|^{2}\right)}
$$

The expressions as follows can be achieved by calculation

$$
\begin{gathered}
p_{r_{i, 1}}=\frac{I_{\max }\left|h_{r_{i, 2}}\right|^{2} a b+\left|g_{r_{i, 2}}\right|^{2} b c}{\left|g_{r_{i, 1}}\right|^{2}\left|h_{r_{i, 2}}\right|^{2} a b-\left|h_{r_{i, 1}}\right|^{2}\left|g_{r_{i, 2}}\right|^{2} a c}+\frac{d_{1}}{\left|g_{r_{i, 1}}\right|\left(\left|g_{r_{i, 1}}\right|^{2}\left|h_{r_{i, 2}}\right|^{2} a b-\left|h_{r_{i, 1}}\right|^{2}\left|g_{r_{i, 2}}\right|^{2} a c\right)} \\
p_{r_{i, 2}}=\frac{I_{\max }}{\left|g_{r_{i, 2}}\right|^{2}}-\frac{\left|g_{r_{i, 1}}\right|^{2}}{\left|g_{r_{i, 2}}\right|^{2}} p_{r_{i, 1}}
\end{gathered}
$$

\section{B. OMP (Optimal approach with Max transmit Power limit of relay) Algorithm}

OMP algorithm is conducting the optimal power allocation under the restraint condition of maximum transmission power of relay; provided that $p_{r_{i, 1}}+p_{r_{i, 2}}=p_{r \max }, p_{r_{i, 1}}\left|g_{r_{i, 1}}\right|^{2}+p_{r_{i, 2}}\left|g_{r_{i, 2}}\right|^{2}=I_{\max }$,namely, when the values of $P_{i_{1}}$ and $P_{i_{2}}$ gradually increase to maximum transmission power of relay, but not reach interference tolerance of primary user therefore, the restraint condition can be simplified as $p_{r_{i, 1}}+p_{r_{i, 2}}=p_{r \max }$

Same as OMI algorithm, provided the variable is

$$
d_{2}=\sqrt{\left(P_{r \max }\left|h_{r_{i, 2}}\right|^{2} a b+b c\right)^{2}-\left(\left|h_{r_{i, 2}}\right|^{2} a b-\left|h_{r_{i, 1}}\right|^{2} a c\right)\left(P_{r a m x}^{2}\left|h_{r_{i, 2}}\right|^{2} a b+P_{r \max }\right)}
$$

By means of Lagrange multiplier [18], the $P_{r_{i, 1}}$ and $P_{r_{i, 2}}$ in the OMP algorithm are

$$
\begin{gathered}
p_{r_{i, 1}}=\frac{P_{r \max }\left|h_{r_{i, 2}}\right|^{2} a b+b c}{\left|h_{r_{i, 2}}\right|^{2} a b-\left|h_{r_{i, 1}}\right|^{2} a c}+\frac{d_{2}}{\left|h_{r_{i, 2}}\right|^{2} a b-\left|h_{r_{i, 1}}\right|^{2} a c} \\
p_{r_{i, 2}}=P_{r \max }-p_{r_{i, 1}}
\end{gathered}
$$

\subsection{Relay Selection Algorithm}

By means of OMI and OMP algorithms, calculate power allocation of all the relays respectively, meanwhile, calculate system throughput in combination with $P_{s}^{*}$ value, and select the relay which can achieve maximum system throughput as the optimal relay. By the expression of $T_{r_{i}}\left(P_{s}, P_{r_{i, 1}}, P_{r_{i, 2}}\right)$,it can be found that the relay model discussed in this article has two aspects that affect system throughput in one aspect, under the condition of cognitive radio, the interference tolerance of primary user should be taken into account for the transmission power range of relay, since it cannot achieve the maximum SINR (Signal to Interference plus Noise Ratio) compared with traditional relay ; In another aspect, the relay 
amplifies the primary user interference and system noise while amplifying the useful signal, and increases interference of cognitive receiving user.

\section{Simulated Result and Comparison}

The article provides OMI and OMP algorithms respectively based on the difference of restraint conditions, and select optimal relay in light of calculating result of such algorithms. The performances of algorithms specified in the article are stated in Figure 2 and 3 respectively. Meanwhile, the following two algorithms are compared on performance.

(1). OEPA (Optimal relay selection with Equal Power Allocation): Select optimal relay and use equal transmission power in sub-channel.

(2). ROPA (Random relay selection with Optimal Power Allocation): Select random relay and use optimal transmission power in sub-channel.

In the article, all the channels are supposed as Rayleigh Fading channels and distributed independently. The path loss factor is 4 .

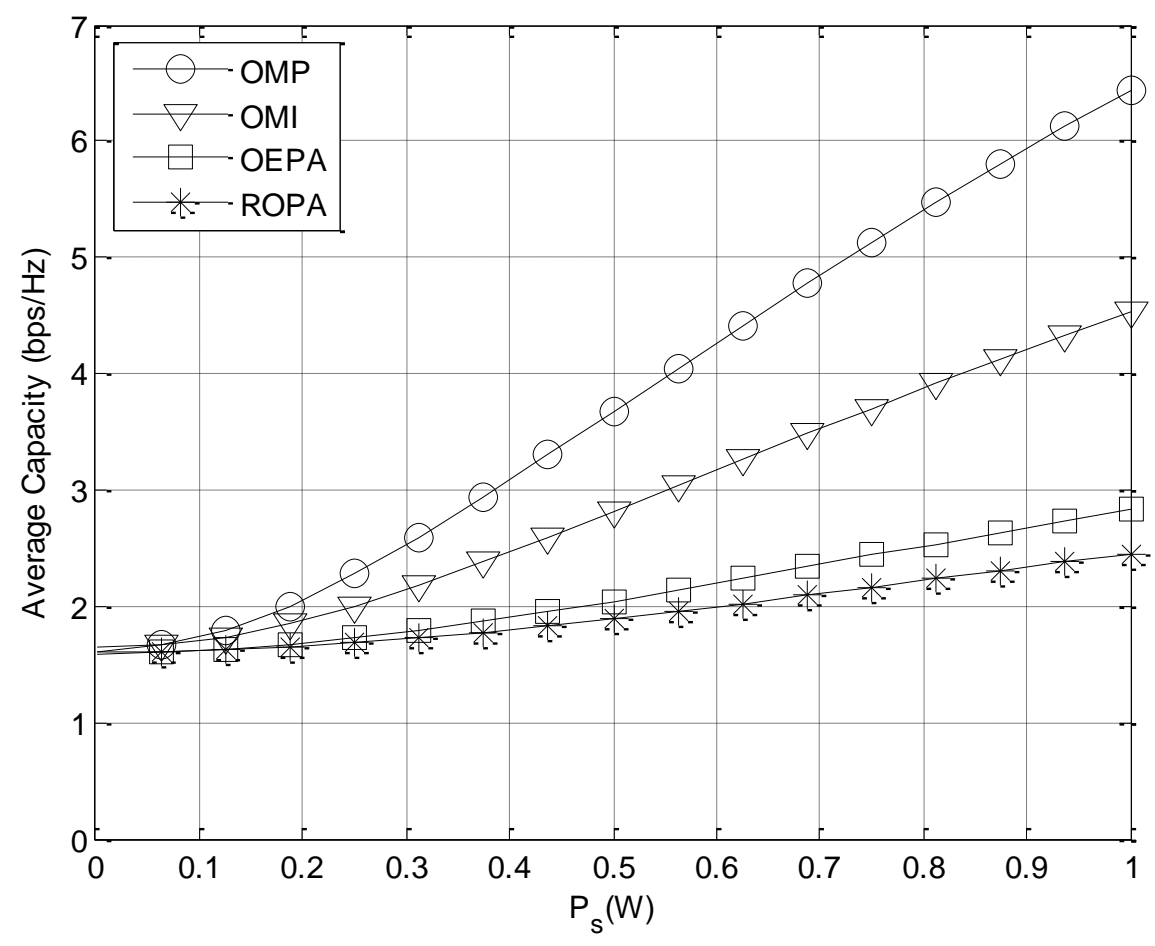

Figure 2. The Relationship between System Throughput and Transmission Power of Cognitive Source

Figure 2 shows the relationship between system throughput and transmission power of cognitive source, provided that the transmission power of selected relay is fixed as $p_{r \max }=1 \mathrm{~W}$, and interference tolerance of primary user is $I_{\max }=0.1 \mathrm{~W}$. There are 10 selectable relays. From the figure, it is shown that the system throughput is monotonically increasing by taking the transmission power of source as variable, and the transmission power of source is 
the decisive factor of system throughput. From the figure, it is shown that compared with OMI algorithm, OMP algorithm can achieve greater system throughput, both of them have the same algorithm mechanism, the only difference is restraint condition. When the interference tolerance of primary user is not taken into account, the relay can play its maximum role so as the maximum system throughput under non-cognitive condition can be achieved, this is the reason why OMP can achieve greater system throughput.

Figure 3 shows the relationship between system throughput and transmission power of relay under interference tolerance of different primary users, provided that there are 10 selectable relays and maximum transmission power of cognitive source is $P_{s}=1 \mathrm{~W}$. From the figure, it is shown that the system throughput is monotonically increasing by taking $P_{r \max }$ transmission power as variable. When $P_{r \max }$ value is less, it has greater impact on system throughput; when $P_{r \max }$ value is more, the system throughput changes slowly and stabilizes at a fixed value due to restraint of interference tolerance of primary user. Therefore, the interference tolerance is decisive factor of system throughput as well.

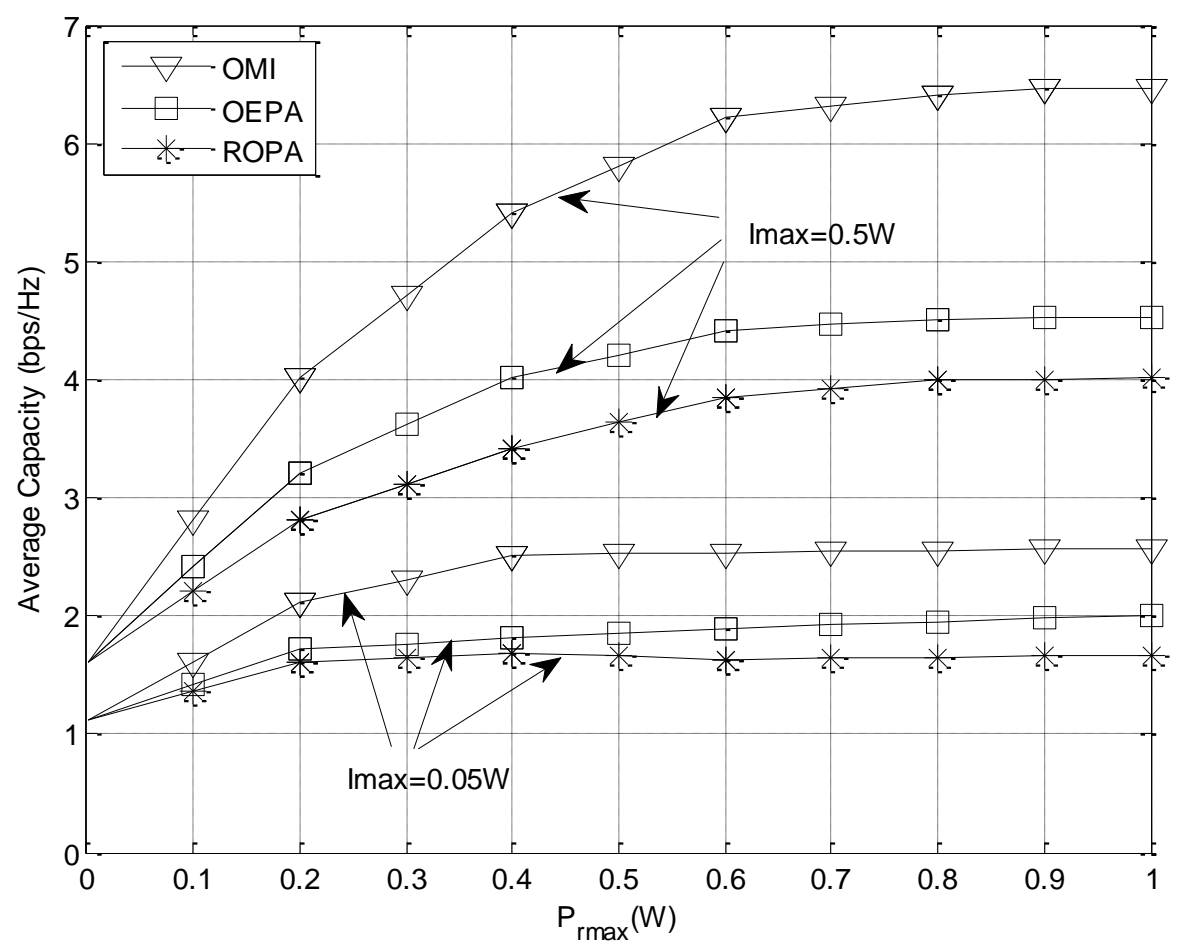

\section{Figure 3. The Relationship between System Throughput and Transmission Power of Relay under Interference Tolerance of Different Primary Users}

\section{Conclusion}

This paper focuses on the optimal relay selection and power allocation issue, which studies the communication between SS and two SDs through single relay in CR system. Under the AF relay mode, according to the different condition, the author puts forward OMP algorithm and OMI algorithm to achieve the optimal relay selection and power allocation; he maximizes 
the system throughput, and solves the energy waste problem. Furthermore, the property of algorithm proposed in this paper is highlighted by simulation and comparison with OEPA and ROPA algorithm.

\section{Acknowledgements}

This research was supported by National Natural Science Foundation and Civil Aviation Ad ministration of China (61071104).

\section{References}

[1] S. Haykin, "Cognitive radio brain-empowered wireless communications", IEEE Journal on Selected Areas in Communications, vol. 23, no. 2, (2005), pp. 201-220.

[2] J. Lee, H. Wang, J. G. Andrews and D. Hong, "Outage probability of cognitive relay networks with interference constraints", IEEE Transactions on Wireless Communications, vol. 10, no. 2, (2011), pp. 390395.

[3] V. Asghari and S. Aissa, "Cooperative relay communication performance under spectrum-sharing resource requirements", in Proc. IEEE International Conference on Communication, (2010) May 1-6, Cape Town, South Africa.

[4] S. Sagong, J. Lee and D. Hong, "Capacity of reactive DF scheme in cognitive relay networks", IEEE Transactions on Wireless Communications, vol. 10, no. 10, (2011), pp. 3133-3138.

[5] D. B. Da Costa and S. Aissa, "Cooperative dual-hop relaying systems with beamforming over Nakagami-m fading channels", IEEE Transactions on Wireless Communications, vol. 8, no. 8, (2009), pp. 3950-3954.

[6] K. Vardhe, D. Reynolds and B. D. Woerner, "Joint power allocation and relay selection for multiuser cooperative communication", IEEE Transactions on Wireless Communications, vol. 9, no. 4, (2010), pp. $1255-1260$.

[7] R.-H. Luo and Y. Zhen, "Power allocation based on the best relay selection in cognitive networks", Chinese Journal of Scientific Instrument, vol. 33, no. 9, (2012), pp. 1931-1937.

[8] Y.-Z. Chu and B.-Y. Zheng, "A Cooperative Transmission Scheme Based on Amplify and Forward in Cognitive Radio Networks", Journal of Electronics \& Information Technology, vol. 33, no. 3, (2012), pp. 509-514.

[9] Y. Li, H.-B. Chen and L. Cong, "Cognitive Relay Selection Algorithm Based on Location and Interference Constraint", Computer Engineering, vol. 38, no. 4, (2012), pp. 7-9.

[10] K. J. Kim, T. Q. Duong and H. V. Poor, "Performance Analysis of Cyclic Prefixed Single-Carrier Cognitive Amplify-and-Forward Relay Systems", IEEE Transactions on Wireless Communications, vol. 12, no. 1, (2012), pp. 195-205.

[11] V. N. Q. Bao, T. Q. Duong and D. Benevides da Costa, "Cognitive Amplify-and-Forward Relaying with Best Relay Selection in Non-Identical Rayleigh Fading”, IEEE Communications letters, vol. 17, no. 3, (2013), pp. 475-478.

[12] A. Bletsas, A. Khisti, D. P. Reed and A. Lippman, “A simple cooperative diversity method based on network path selection”, IEEE Journal on selected Areas in Communications, vol. 24, no. 3, (2006), pp. 659-672.

[13] J. Jia, J. Zhang and Q. Zhang, “Cooperative relay for cognitive radio networks", IEEE INFOCOM, (2009) April 19-25, Rio de Janeiro, Brazil.

[14] L. Li, X. Zhou, H. Xu, G. Y. Li, D. Wang and A. Soong, "Simplified relay selection and power allocation in cooperative cognitive radio systems", Transactions on Wireless Communications, vol. 10, no. 1, (2011), pp. 33-36.

[15] Q. Cao, Y. Jing and H. Zhao, "Power Allocation in Multi-User Wireless Relay Networks through Bargaining", IEEE Transactions on Wireless Communications, vol. 12, no. 6, (2013), pp. 2870-2882.

[16] M. Alam, J. Mark and X. Shen, "Relay Selection and Resource Allocation for Multi-User Cooperative OFDMA Networks", IEEE Transactions on Wireless Communications, vol. 12, no. 5, (2013), pp. 2193-2205.

[17] G. A. S. Sidhu, F. Gao, W. Wang and W. Chen, "Resource Allocation in Relay-Aided OFDM Cognitive Radio Networks", IEEE Transactions on Vehicular Technology, vol. 62, no. 8, (2013), pp. 3700-3710.

[18] S. Boyd and L. Vandenberghe, "Convex Optimization”, Cambridge University Press, New York (2004). 


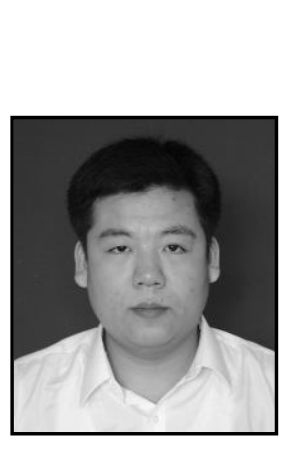

\section{Authors}

Guanglong Yang, he received the B.S. and the M.S. degrees from Northeast Agricultural University, Harbin, China, in 2003 and 2006, respectively. He is currently working toward the Ph.D. degree with the School of Electronics and Information Engineering, Harbin Institute of Technology. From 2007 to 2012, he was with Qiaohang Communication Company, Harbin, where he worked on the research and development of the digital trunking system. His research interests include power control and spectrum allocation for cognitive radio networks.

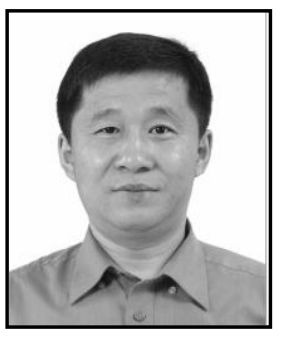

Xiao Wang, he received the B.S. degrees from Xi'an Jiao Tong University, Xianin, China, in 1988, received the M.S. degrees from Harbin Institute of Technology, Harbin, China, in 2005. He worked for Department of Electronics and Communication Engineering HIT as an associate professor and master's tutor since 2002. Now he is member of Heilongjiang Electronics Engineering Society. Ass Prof. Wang's research areas include the trunked mobile communication system, broadband multimedia mobile communication system, wireless network and digital transmission technology and other aspects of the scientific research and teaching work.

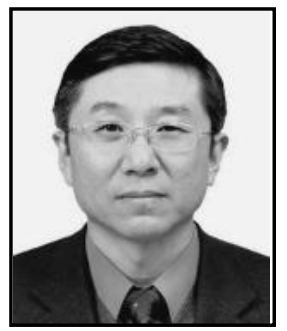

Xuezhi Tan, he received the B.S., M.S., and Ph.D. degrees from Harbin Institute of Technology, Harbin, China, in 1982, 1986, and 2005, respectively. From October 1988 to March 1990, he was a Visiting Scholar with Kyoto University, Kyoto, Japan. $\mathrm{He}$ is currently a Professor with the School of Electronics and Information Engineering, Harbin Institute of Technology. He is a Senior Member of the Chinese Institute of Electronics and the Chinese Institute of Communication. He had published more than 70 papers in international journals. His research interests include wireless communications, digital trunking communication, and cognitive radio. 
International Journal of Future Generation Communication and Networking Vol. 7, No. 6 (2014) 